\title{
Selective Recognition of a Single HIV-1 G-Quadruplex by Ultrafast Small-Molecule Screening
}

\author{
Matteo Scalabrin, ${ }^{\S}$ Matteo Nadai, ${ }^{\S}$ Martina Tassinari, Sara Lago, Filippo Doria, Ilaria Frasson, \\ Mauro Freccero, and Sara N. Richter*
}

Cite This: Anal. Chem. 2021, 93, 15243-15252

Read Online

ABSTRACT: G-quadruplexes (G4s) are implicated in pathological processes such as cancer and infective diseases. Their targeting with G4-ligands has shown therapeutic capacity. Most of the current G4-ligands are planar molecules, do not discriminate among G4s, and have poor druglike properties. The available methods to identify compounds selective for one single G4 are often time-consuming. Here, we describe the development, validation, and application of an affinity-selection mass spectrometry method that employs unlabeled G4 oligonucleotides as targets and allows testing of up to 320 unmodified small molecules in a single tube. As a proof of concept, this method was applied to screen a library of 40000 druglike molecules against two G4s,

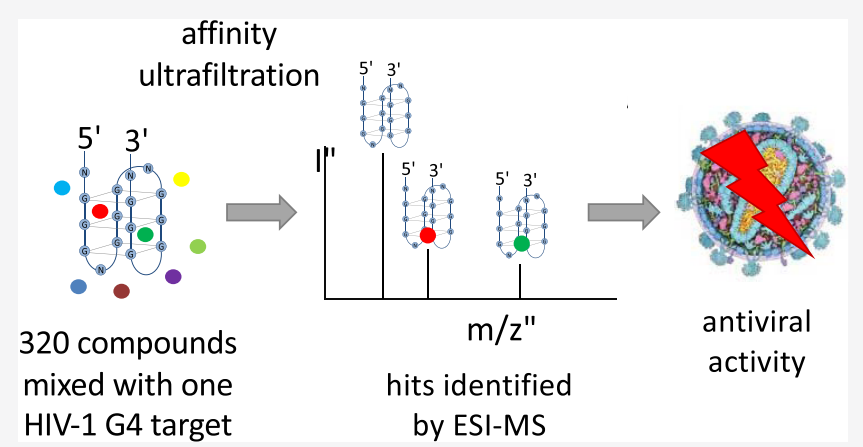
transcriptional regulators of the HIV-1 LTR promoter. We identified nonplanar pyrazolopyrimidines that selectively recognize and stabilize the major HIV-1 LTR G4 possibly by fitting and binding through H-bonding in its unique binding pocket. The compounds inhibit LTR promoter activity and HIV-1 replication. We propose this method to prompt the fast development of new G4-based therapeutics.

G-quadruplexes (G4s) are noncanonical nucleic acid secondary structures that are increasingly being recognized as key regulators of important biological and pathological processes in humans, ${ }^{1,2}$ including cancer, ${ }^{3,4}$ neurodegenerative disorders, ${ }^{5}$ and infective diseases caused by parasites, prokaryotes, and viruses. $^{6-8}$ HIV-1, the lentivirus responsible for the acquired immune deficiency syndrome (AIDS), ${ }^{9}$ possesses an ssRNA genome that, once retrotranscribed into DNA by the viral reverse transcriptase enzyme, integrates into the host cell chromosome. In the long terminal repeat (LTR) promoter of the integrated HIV-1 genome, overlapping G4s were shown to control viral transcription and be modulated by the interaction with cellular proteins. ${ }^{10-13}$ It follows that G4s represent potential targets for the development of therapies against critical human diseases. ${ }^{1,14}$ Indeed, promising therapeutic activities of G4-ligands, compounds able to selectively recognize G4s over dsDNA, have been reported. ${ }^{8,15-17}$ Different strategies have been developed to implement selectivity, ${ }^{15,18-24}$ with the identification/development of selective compounds being in general a long and/or cumbersome process.

Electrospray ionization (ESI) mass spectrometry (MS) is a powerful and sufficiently gentle technique to investigate noncovalent nucleic acid-ligand binding. ${ }^{25-28}$ To overcome the incompatibility of the high $\mathrm{K}^{+}$concentration required for G4 folding with MS analysis, buffers that allow both G4folding and MS analysis have been recently developed. ${ }^{29-34}$
Here, we present an affinity-ultrafiltration MS (AUF-MS)based method to afford the ultrafast high-throughput screening (HTS) of small molecules against G4s. We first validated the method using known G4 ligands; next, as a proof of concept, we employed it to screen a 40000 -compound library against two G4-folding sequences present in the LTR promoter of HIV-1 (Table S1). ${ }^{10,35,36}$ The best-recovered molecule had a nonplanar core, specifically recognized one of the two LTR G4s, i.e., LTR-III, at the unique junction between the G4 and the stem loop and displayed anti-HIV-1 activity.

\section{EXPERIMENTAL SECTION}

Compounds. The test compounds proflavine hydrochloride, 9-aminoacridine, 9(10H)-acridanone, 1,5-diaminoanthraquinone, and doxorubicin hydrochloride were purchased from Sigma-Aldrich (Saint Louis, MO). BRACO-19 (B19) was supplied by Endotherm Life Science, Saarbrücken, Germany. NDI-morph, NDI-Gly, and NDI-alk have been previously published. ${ }^{37-39}$ The DiverSet small-molecule library was

Received: September 29, 2020

Accepted: October 18, 2021

Published: November 11, 2021 
purchased from ChemBridge (San Diego, CA) and consists of 40000 compounds displaying the following physiochemical properties: molecular weight $(\mathrm{MW}) \leq 500 \mathrm{Da}$, partition coefficient for $n$-octanol/water $(\operatorname{clog} \mathrm{P}) \leq 5$, topological polar surface area $(\mathrm{tPSA}) \leq 100 \AA^{2}$, rotatable bonds $\leq 8$, hydrogenbond acceptors $\leq 10$, and hydrogen-bond donors $\leq 5$. Library hit compounds and analogues were purchased in lyophilized form and dissolved in dimethyl sulfoxide (DMSO) to the final concentration of $10 \mathrm{mM}$. See the Excel file in Supporting Information for the list of compounds and their properties.

Circular Dichroism (CD) Analysis. Experiments were performed using a Chirascan-Plus (Applied Photophysics, Leatherhead, U.K.) equipped with a Peltier temperature controller using a quartz cell of $5 \mathrm{~mm}$ path length. G4 folding oligonucleotides were diluted to a final concentration of $2 \mu \mathrm{M}$ in $100 \mathrm{mM} \mathrm{KCl}$ and $10 \mathrm{mM}$ lithium cacodylate (SigmaAldrich) buffer at $\mathrm{pH} 7.4$ (CD buffer). After annealing (5 min at $95{ }^{\circ} \mathrm{C}$ ), DNA samples were gradually cooled down to room temperature (rt), and, where specified, the tested compounds in $\mathrm{CD}$ buffer were added at a final concentration of $10 \mu \mathrm{M}$. Alternatively, oligonucleotides $(2 \mu \mathrm{M})$ in $1 \mathrm{mM} \mathrm{KCl}$ and 150 $\mathrm{mM}$ hexafluoroisopropanol (HFIP) (Acros, Pittsburgh, PA) neutralized to $\mathrm{pH} 7.4$ with triethylamine (TEA, final concentration $2.7 \mathrm{mM}$ ) were annealed by heating at $95{ }^{\circ} \mathrm{C}$ for $5 \mathrm{~min}$, gradually cooled to $\mathrm{rt}$, added to $20 \%$ isopropanol (IPA), and incubated overnight at $4{ }^{\circ} \mathrm{C}$ (the final composition of this buffer, from now on referred to as MS buffer, is: $0.8 \mathrm{mM}$ $\mathrm{KCl}$, HFIP (120 mM)/TEA pH 7.4, 20\% IPA). Thermal unfolding analyses were recorded from 230 to $320 \mathrm{~nm}$ over a temperature range of $20-90{ }^{\circ} \mathrm{C}\left(5^{\circ} \mathrm{C} / \mathrm{min}\right)$. Observed ellipticities were converted to mean residue ellipticity $(\theta)=$ deg $\times \mathrm{cm}^{2} \times \mathrm{dmol}^{-1}$ (mol ellip). $T_{\mathrm{m}}$ values were calculated according to the Van't Hoff equation, applied for a two-state transition, assuming that the heat capacities of the folded and unfolded states are equal. ${ }^{40}$

Affinity-Ultrafiltration Mass Spectrometry Analysis. Mass spectrometry samples were prepared mixing the tested oligonucleotides $(20 \mu \mathrm{L}$ of a $5 \mu \mathrm{M}$ solution annealed in MS buffer as described in CD experiments) with 5-fold excess of the tested compound(s). Compound library mixtures (stock concentration, $500 \mu \mathrm{M}$ of each compound) were prepared by mixing 320 compounds in water using an automatic liquid handling workstation Caliper Zephyr (Caliper LifeSciences, Hopkinton, MA); each compound/mixture was diluted in MS buffer before incubation overnight at $4{ }^{\circ} \mathrm{C}$ with the tested G4 in a final volume of $500 \mu \mathrm{L}$. Unbound compounds were removed by three steps of ultrafiltration in a Millipore (North Bend, $\mathrm{OH}$ ) centrifugal filter with a $3 \mathrm{kDa}$ molecular weight cutoff, and $500 \mu \mathrm{L}$ of MS buffer at $4{ }^{\circ} \mathrm{C}$ was refilled after the first and second steps. Each wash/ultrafiltration step was performed in $40 \mathrm{~min}$, for a total of $2 \mathrm{~h}$ for the whole wash/ ultrafiltration procedure. The wash/ultrafiltration time was kept constant between samples. The concentrated G4 solution obtained after the third step $(40 \mu \mathrm{l})$ was directly analyzed by mass spectrometry. Samples were analyzed by direct infusion electrospray ionization (ESI) on a Xevo G2-XS QTOF mass spectrometer (Waters, Manchester, U.K.). This is a highresolution instrument that allowed us to visualize the isotopic pattern, identify the charge state, and therefore unambiguously calculate the neutral mass of the detected species. The injection was automatically performed by an Agilent 1290 Infinity HPLC (Agilent Technologies, Santa Clara, CA) equipped with an autosampler; the carrying buffer was HFIP
$(120 \mathrm{mM}) /$ TEA pH 7.4 with $20 \%$ IPA. A volume of $5 \mu \mathrm{L}$ of each sample was typically injected. In all experiments, ESI source settings were: electrospray capillary voltage, $1.8 \mathrm{kV}$; source and desolvation temperatures, 45 and $65{ }^{\circ} \mathrm{C}$, respectively; sampling cone voltage, $65 \mathrm{~V}$. All of these parameters ensured minimal DNA complexes fragmentation. The instrument was calibrated using a $2 \mathrm{mg} / \mathrm{mL}$ solution of sodium iodide in $50 \%$ IPA. The additional use of the LockSpray during analysis provided a typical $<5$ ppm mass accuracy. The internal standard LockSpray consisted of a solution of leu-enkephalin $(1 \mu \mathrm{g} / \mathrm{mL})$ in acetonitrile/water (50:50, v/v) containing $0.1 \%$ formic acid. The $\%$ of the ratio bound G4 over total G4 was calculated according to the following formula: [bound G4/G4 (\%) $=\left(\sum \mathrm{G} 4 \mathrm{~b} /\left(\sum \mathrm{G} 4 \mathrm{f}+\right.\right.$ $\left.\left.\sum \mathrm{G} 4 \mathrm{~b}\right)\right) \times 100$ ], where $\mathrm{G} 4 \mathrm{~b}$ is the peak intensity of bound $\mathrm{G} 4$ DNA and G4f is the peak intensity of free G4 DNA obtained after the deconvolution with the software BiopharmaLynx. The mass shift was calculated manually from the monoisotopic mass visualized in the mass spectra or from the deconvoluted average masses calculated by BiopharmaLynx.

Molecular Modeling. The NMR structure of LTR-III Gquadruplex (PDB 6H1K) was used for the docking study. ${ }^{36}$ The structure of the pyrazolopyrimidines analyzed was constructed and optimized with Gaussian 09 using the HF/ 6-31G** basis set. Receptor grid was generated using Receptor grid generation in the Glide application (Glide, version 6.9, Schrödinger, LLC, and New York) of Maestro (Schrödinger, LLC, New York, NY, 2019-3). Once the receptor grid was generated, the ligands were docked to the G4 target using Glide version 6.9 (Grid based Ligand Docking with Energetics) docking protocol. The ligands were docked using the IFD protocol. The docked conformers were evaluated using Glide (G) Score. The G Score was calculated as follows: $\mathrm{G}$ Score $=a^{*} \mathrm{vdW}+b^{*} \mathrm{Coul}+\mathrm{Lipo}+\mathrm{Hbond}+$ Metal + BuryP + RotB + Site, where vdW denotes van der Waals energy, Coul denotes Coulomb energy, Lipo denotes lipophilic contact, HBond indicates hydrogen bonding, Metal indicates metal binding, BuryP indicates penalty for buried polar groups, RotB indicates penalty for freezing rotatable bonds, Site denotes polar interactions in the active site, and $a=0.065$ and $b=$ 0.130 are coefficients of $\mathrm{vdW}$ and Coul, respectively. Visualization of the docked pose was obtained using UCSF Chimera molecular graphics program.

Binding Affinity. To determine $K_{\mathrm{D}}$ of J20 ligand to LTRIII, mass spectrometry analysis was performed both on equimolar mixtures of LTR-III + J20 $(2,4$, and $10 \mu \mathrm{M})$ and on a mixture of $2 \mu \mathrm{M}$ reference dT $6+5 \mu \mathrm{M}$ LTR-III +5 or 10 $\mu \mathrm{M} \mathrm{J} 20$. The peak areas were used to calculate the concentration ratios, as previously reported, ${ }^{41}$ using the following equations

$$
\begin{aligned}
& {[\mathrm{LTR}-\mathrm{III}]_{\mathrm{free}}=C_{0} \times A(\mathrm{LTR}-\mathrm{III})^{n-} /\left(A(\mathrm{LTR}-\mathrm{III})^{n-}\right.} \\
& \left.+A(\mathrm{LTR}-\mathrm{III}+\mathrm{J} 20)^{n-}\right) \\
& {[\mathrm{LTR}-\mathrm{III}+\mathrm{J} 20]} \\
& =C_{0} \times A(\mathrm{LTR}-\mathrm{III}+\mathrm{J} 20)^{n-} /\left(A(\mathrm{LTR}-\mathrm{III})^{n-}\right. \\
& \left.+A(\mathrm{LTR}-\mathrm{III}+\mathrm{J} 20)^{n-}\right) \\
& {[\mathrm{J} 20]_{\text {free }}=[\mathrm{J} 20]_{\text {tot }}-[\mathrm{LTR}-\mathrm{III}+\mathrm{J} 20]} \\
& K_{\mathrm{d}}=[\mathrm{J} 20]_{\text {free }} \times[\mathrm{LTR}-\mathrm{III}]_{\text {free }} /[\mathrm{LTR}-\mathrm{III}+\mathrm{J} 20]
\end{aligned}
$$


where $[\mathrm{LTR}-\mathrm{III}]_{\text {free }}$ and $[\mathrm{J} 20]_{\text {free }}$ are the concentrations of the unbound LTR-III and J20, respectively; $[\mathrm{J} 20]_{\text {tot }}$ is the total concentration of J20; [LTR-III + J20] is the concentration of J20 bound to LTR-III; $C_{0}$ is the starting LTR-III concentration; $A(\mathrm{LTR}-\mathrm{III})^{n-}$ is the peak area of the oligonucleotide alone at charge state $n-$; and A(LTR-III + $\mathrm{J} 20)^{n-}$ is the peak area of J20 bound to LTR-III at charge state $n-$. Peak areas were calculated using MassLynx 4.1 software (Waters), after processing steps consisting of smoothing, background subtraction, and conversion to centroid.

NMR Spectroscopy. NMR experiments were performed on a NEO $700 \mathrm{MHz}$ Avance (Bruker) spectrometer, equipped with $\mathrm{He}$ cryoprobe. ${ }^{1} \mathrm{H}$ NMR spectra were recorded at $25{ }^{\circ} \mathrm{C}$.

Luciferase Reporter Assay. Luciferase activity of HIV-1 LTR was assessed in human embryonic kidney 293T (HEK 293T) as previously reported. ${ }^{10}$ Details are provided in Supporting Information.

Cell Viability Assay. Effects on cell viability were determined by 3-(4,5-dimethylthiazol-2-yl)-2,5-diphenyltetrazolium bromide (MTT) assay. HEK 293T cells were plated (6 $\times 10^{3}$ ) into 96-microwell plates to a final volume of $100 \mu \mathrm{L}$ and allowed an overnight period for attachment. The following day, the tested compounds were added to each well and tested in triplicate. Control cells were treated in the exact same conditions. Cell survival was evaluated by MTT assay, $48 \mathrm{~h}$ after treatment: $10 \mu \mathrm{L}$ of freshly dissolved solution of MTT (5 $\mathrm{mg} / \mathrm{mL}$ in phosphate-buffered saline, PBS) was added to each well, and after $4 \mathrm{~h}$ of incubation, MTT crystals were solubilized in solubilization solution (10\% sodium dodecyl sulfate (SDS) and $0.01 \mathrm{M} \mathrm{HCl}$ ). After overnight incubation at $37{ }^{\circ} \mathrm{C}$, absorbance was read at $540 \mathrm{~nm}$. The percentage of cell survival was calculated as follows: cell survival $=\left(A_{\text {well }}-A_{\text {blank }}\right) /$ $\left(A_{\text {control }}-A_{\text {blank }}\right) \times 100$, where blank denotes the medium without cells. Each experiment was repeated at least three times. MTT assay was performed twice, in triplicate.

Taq Polymerase Stop Assay. The DNA primer (Table S1) was $5^{\prime}$-end labeled with $\left[\gamma_{-}{ }^{32} \mathrm{P}\right]$ ATP using T4 polynucleotide kinase (Thermo Scientific, Milan, Italy) at $37^{\circ} \mathrm{C}$ for 30 min. The labeled primer (final concentration, $72 \mathrm{nM}$ ) was annealed to the templates (final concentration, $36 \mathrm{nM}$ ) (Table $\mathrm{S} 1)$ in lithium cacodylate buffer $(10 \mathrm{mM}, \mathrm{pH} 7.4)$ in the presence of $\mathrm{KCl} 20 \mathrm{mM}$ by heating at $95{ }^{\circ} \mathrm{C}$ for $5 \mathrm{~min}$ and gradually cooling to $\mathrm{rt}$ to allow both primer annealing and G4 folding. Where specified, samples were incubated with J20, $\mathrm{J} 20 \mathrm{~B}$, or B08 $(1-4 \mu \mathrm{M})$ and primer extension was performed with $2 \mathrm{U} /$ reaction of AmpliTaq Gold DNA polymerase (Applied Biosystem, Carlsbad, CA) at $42{ }^{\circ} \mathrm{C}$ for $30 \mathrm{~min}$. Reactions were stopped by ethanol precipitation, and primer extension products were separated on a $16 \%$ denaturing gel and visualized by phosphorimaging (Typhoon FLA 9000, GE Healthcare, Milan, Italy). Markers were prepared based on Maxam \& Gilbert sequencing by polymerase chain reaction (PCR) reaction with ${ }^{32} \mathrm{P}$-labeled primer. PCR products were treated with formic acids for $5 \mathrm{~min}$ at $25{ }^{\circ} \mathrm{C}$ and then with piperidin for $30 \mathrm{~min}$ at $90{ }^{\circ} \mathrm{C}$.

Luciferase Reporter Assay. The wild-type (WT) HIV-1 LTR region (corresponding to segment $-381 /+83$ in the HIV1 genome) was inserted into the promoterless luciferase reporter vector pGL4.10-Luc2 (Promega Italia, Milan, Italy) to make pGL4.10-LTR. HEK 293T cells sustain all viral steps except for virion attachment and entry as they lack cell receptors recognized by HIV-1. They can be transfected with the HIV-1 proviral genome to produce fully infectious viral particles, indicating that their cytoplasmic/nuclear protein makeup sustains viral transcription and replication. HEK 293T cells were seeded in 24-well plates $\left(0.75 \times 10^{5}\right.$ cells/well $)$. Cells were transfected $24 \mathrm{~h}$ post-seeding with pGL4.10-LTR (100 ng/well) using Lipofectamine 3000 transfection reagent (Invitrogen, Thermo Fisher Scientific, Milan, Italy), according to the manufacturer's protocol. After $90 \mathrm{~min}$, the transfection medium was removed and the cells were washed and treated with increasing concentrations $(0.15-5 \mu \mathrm{M})$ of the indicated compounds for $24 \mathrm{~h}$. Control cells were treated with dimethyl sulfoxide (DMSO), as it was the solvent of the administered compounds. Luciferase activity was measured using the britelite plus Reporter Gene Assay System (PerkinElmer, Inc., Milan, Italy) in a Victor X2 multilabel plate reader (PerkinElmer, Inc., Milan, Italy). Cells were lysed in lysis buffer ( $1 \times$ PBS, $1 \%$ TRITON X-100), and protein concentration was determined. Luciferase signals were normalized to total protein content, according to the manufacturer's protocol. All experiments were performed twice and in duplicate.

Antiviral Assay. TZM-bl cell line was provided by NIH AIDS Research Program. TZM-bl is a HeLa cell line stably expressing large amounts of CD4 and CCR5 and containing integrated copies of the reporter genes for firefly luciferase and Escherichia coli $\beta$-galactosidase under control of the HIV-1 promoter. HEK 293T (Human Embryonic Kidney 293T) cell line, a highly transfectable derivative of human embryonic kidney 293 cells, was provided by ATCC. Both cell lines were maintained in Dulbecco's modified Eagle's medium (DMEM) (Gibco, Life Technologies, Monza, Italy) supplemented with $10 \%$ heat-inactivated fetal bovine serum (FBS) (Gibco, Life Technologies, Monza, Italy) at $37{ }^{\circ} \mathrm{C}$ in a humidified $\mathrm{CO}_{2}$ controlled atmosphere. The HIV-1 strain NL4-3 viral stock was prepared by transfection of HEK 293T cells with wild-type X4 proviral genome (NIH AIDS Research and Reference Reagent Program), using CalPhos mammalian transfection kit (Clontech Laboratories, Santa Clara, CA) according to the manufacturer's protocol. Viral particles in supernatants were collected and titrated by the Reed and Muench method. HIV-1 infectivity was measured in TZM-bl cells, in which the HIV infection drives transcription of the HIV-1 LTR-luciferase reporter gene construct, and thus, it is possible to evaluate the antiviral effect of test compounds following luciferase expression. TZM-bl cells were seeded in 96-well plates (10000 cells/well) and grown overnight to permit adherence prior to treatment and viral infection. The 50\% cytotoxic concentration $\left(\mathrm{CC}_{50}\right)$ is defined as the concentration of compound that reduces the absorbance of the mock-infected cells by $50 \%$. The $50 \%$ effective concentration $\left(\mathrm{EC}_{50}\right)$ is defined as the concentration of compound that inhibits $50 \%$ LTR-Luciferase signal. The selectivity index (SI) is the relative effectiveness of the tested compound in inhibiting viral replication compared to inducing cell death $\left(\mathrm{CC}_{50} / \mathrm{EC}_{50}\right)$.

\section{RESULTS}

Setup and Validation of the G4-Ligand Screening. We designed a high-throughput screening (HTS) protocol based on an affinity-ultrafiltration mass spectrometry (AUF-MS) approach $^{42}$ (Figure 1) consisting of: (1) incubation of the test molecules with the target G4. This step was performed in MScompatible buffer that had been previously specifically designed to maintain G4 folding and ligand binding while allowing MS analysis; ${ }^{32}$ (2) removal of the unbound molecules 


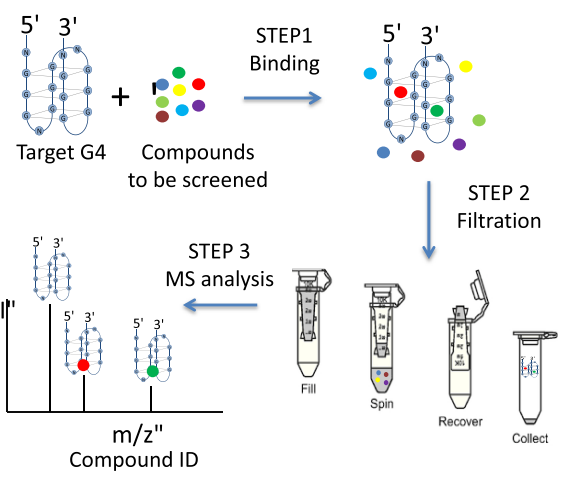

Figure 1. Scheme of the described AUF-MS HTS screening procedure.

(the molecular weight (MW) of which normally ranges between 0.3 and $0.6 \mathrm{kDa}$ ), using a $3 \mathrm{kDa} \mathrm{MW}$ cutoff filter, while preserving the G4/bound molecule complexes, since G4 sequences usually have MW higher than $6 \mathrm{kDa}$; and (3) MS analysis of the G4/ligand complexes. Two options are available here: the indirect method that after filtration requires to dissociate the bound molecules and further filtrate them to be analyzed in the absence of the target biomolecule, or the direct method that analyzes the G4/ligand complexes that remained in the supernatant. We tested both approaches. We found that a major drawback of the indirect method was retention of the unbound molecules on the filter: this could highly increase the number of false-positive hits. For example, the well-known BRACO-19 (B19) G4 ligand at $50 \mu \mathrm{M}$ in HFIP (120 mM)/ TEA $\mathrm{pH} 7.4$ buffer was mainly retained on the filter, as detected by the different molar absorption of B19 in the supernatant and flow-through after filtration and by the yellow color of the filter (Figure S1A). This effect was partially mitigated by addition of isopropanol (IPA) to the MScompatible buffer that, while promoting G4 folding, ${ }^{32}$ minimized B19 stacking onto the membrane (Figure S1B). However, since different molecules could display different filter retention properties, we switched to the direct method. Normally, the main limitation of this strategy is the possibility to detect the complexes of the target and bound molecule/s by MS. However, the previously described optimization of the buffer conditions and MS settings ${ }^{32}$ made this approach feasible.

The AUF-MS HTS method was employed against LTR-III and LTR-IV, two G4-folding sequences present in the LTR
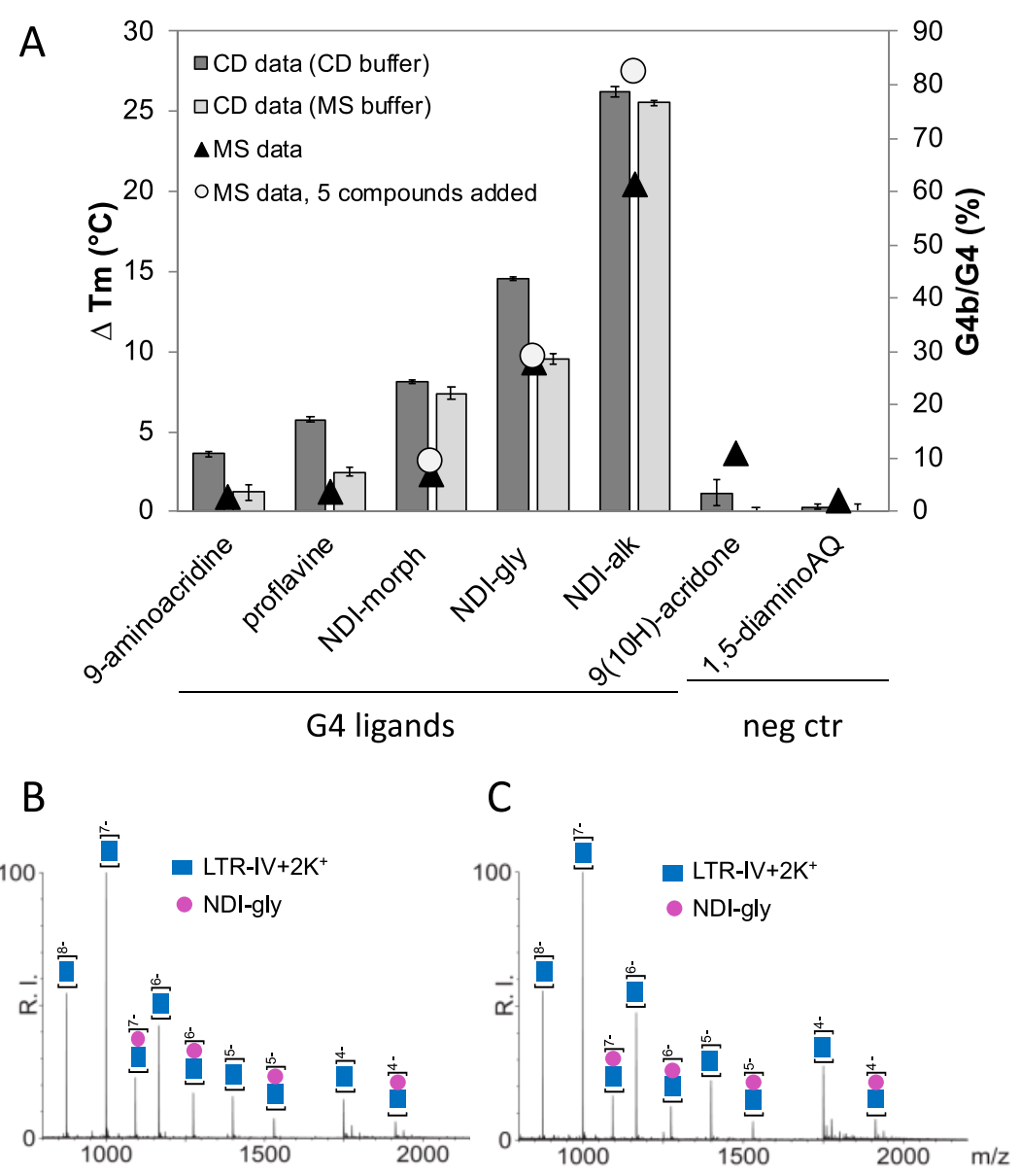

Figure 2. Comparison of known G4-ligand binding by AUF-MS and standard CD analysis. (A) Stabilization $\left(\Delta T_{\mathrm{m}}\right)$ of LTR-IV G4 by each test compound was measured by $\mathrm{CD}$ in $\mathrm{CD}$ buffer $(10 \mathrm{mM}$ lithium cacodylate $\mathrm{pH} 7.4,100 \mathrm{mM} \mathrm{KCl}$ ) (dark gray bars) and in MS buffer (HFIP 120 $\mathrm{mM} /$ TEA pH 7.4, $0.8 \mathrm{mM} \mathrm{KCl})$ (light gray bars). Binding ((G4b/G4)\%) to LTR-IV of single test compounds (black triangles) or in the presence of four other low binding compounds (proflavine, 9-aminoacridine, 9(10H)-acridone, and 1,5-diaminoanthraquinone) (white circles) was measured by MS in MS buffer. Bars refer to $\Delta T_{\mathrm{m}}\left({ }^{\circ} \mathrm{C}\right)$ left $Y$ axis; triangles and circles refer to G4b/G4 (\%) right $Y$ axis. (B, C) MS spectra of LTRIV (squares) incubated with NDI-gly (circles) and the four weak/nonbinding compounds (B) or 320 library compounds (C). Numbers above peaks represent the charge state. 
Table 1. Positive Hits Identified from the Screening of 40000 Library Compounds with the Described AUF-MS Method ${ }^{a}$

\begin{tabular}{|c|c|c|c|c|c|c|c|c|}
\hline \multirow{3}{*}{ hit } & \multicolumn{6}{|c|}{ MS analysis } & \multicolumn{2}{|c|}{$\mathrm{CD}$ analysis } \\
\hline & $\begin{array}{c}\text { monoisotopic mass } \\
(\mathrm{amu})\end{array}$ & mass shift (amu) & $\begin{array}{c}\mathrm{G} 4 \mathrm{~b} / \mathrm{G} 4 \\
(\%)^{\mathrm{S}}\end{array}$ & $\begin{array}{c}\mathrm{G} 4 \mathrm{~b} / \mathrm{G} 4 \\
(\%)^{*}\end{array}$ & $\begin{array}{c}\mathrm{G} 4 \mathrm{~b} / \mathrm{G} 4 \\
(\%)^{\$}\end{array}$ & $\begin{array}{l}\mathrm{G} 4 \mathrm{~b} / \mathrm{G} 4 \\
(\%)^{*}\end{array}$ & $\Delta T_{\mathrm{m}} 265 \mathrm{~nm}$ & $\begin{array}{c}\Delta T_{\mathrm{m}} 284 \mathrm{~nm} \\
\left({ }^{\circ} \mathrm{C}\right)\end{array}$ \\
\hline & & & \multicolumn{2}{|c|}{ LTR-IV } & \multicolumn{2}{|c|}{ LTR-III } & LTR-IV & LTR-III \\
\hline F18 & 328.212 & $328.123 \pm 0.153$ & $17.6 \pm 3.6$ & $20.3 \pm 2.2$ & $<10$ & $<10$ & $2.0 \pm 0.2$ & $<1$ \\
\hline $\mathrm{K} 23$ & 376.154 & $376.100 \pm 0.024$ & $21.3 \pm 0.9$ & $19.6 \pm 0.3$ & $<10$ & $<10$ & $<1$ & $<1$ \\
\hline $\mathrm{J} 20$ & 363.181 & $363.23 \pm 0.071$ & $<10$ & $<10$ & $18.3 \pm 4.6$ & $35.5 \pm 3.3$ & $<1$ & $3.9 \pm 0.5$ \\
\hline N13 & 367.172 & $367.684 \pm 0.023$ & $<10$ & $<10$ & $13.6 \pm 3.6$ & $22.4 \pm 4.3$ & $<1$ & $1.6 \pm 0.4$ \\
\hline
\end{tabular}

${ }^{a}$ \$G4b/G4 \% measured in the mixture of 320 compounds; *G4b/G4\% measured when only the single positive hit was present.

promoter of HIV-1 (Table S1) ${ }^{10}$ that folds into different G4 conformations. In particular, LTR-IV is a parallel stranded G4 with a single nucleotide bulge, ${ }^{35}$ while LTR-III is a hybrid type G4 with a stem loop ${ }^{36}$ (Figure S2). These were shown to control viral transcription, be modulated by cellular proteins, and reduce viral replication when stabilized by known G4 ligands. ${ }^{12,13,43,44}$

To validate our protocol, we initially assessed by circular dichroism (CD) the binding of known G4-stabilizing ligands and two negative controls (Figure S3) to the LTR-IV G4 target. Binding was evaluated by $\mathrm{CD}$ spectral behavior and melting temperature $\left(T_{\mathrm{m}}\right)$ that normally increases upon binding. $\mathrm{CD}$ analysis was performed in either $\mathrm{CD}(10 \mathrm{mM}$ lithium cacodylate $\mathrm{pH} 7.4, \mathrm{KCl} 100 \mathrm{mM}$ )- or MS (120 mM HFIP/TEA pH 7.4, 20\% IPA, KCl $0.8 \mathrm{mM}$ )-optimized buffers. LTR-IV $(2 \mu \mathrm{M})$ melting temperatures were 50.6 and $47.9^{\circ} \mathrm{C}$ in $\mathrm{CD}$ and $\mathrm{MS}$ buffers, respectively. $\Delta T_{\mathrm{m}}$ values upon compound addition $(10 \mu \mathrm{M})$ were slightly higher when measured in CD buffer than in MS buffer (Figure 2A and Table S2), but the $\Delta T_{\mathrm{m}}$ trend among the different compounds was maintained. Compound binding to LTR-IV was next measured by MS in MS buffer. The amount of bound G4 over total amount of G4 (G4b/G4) paralleled compound stability measured by CD (Figure 2A and Table S2). The ultrafiltration and wash steps had a minor impact on the G4-compound complex, as the amount of the G4-ligand complex remained about $90 \%$ of the initial complex with different ligands and targets (Figure S4). We next set up a small-scale screening with test mixtures containing a good G4 binder and 4 weak/ nonbinding compounds. These mixtures were incubated with the folded LTR-IV: solutions were submitted to three steps of ultrafiltration to remove the unbound compounds (Figures S5-S7). A straightforward correlation between $\Delta T_{\mathrm{m}}$ and $\mathrm{G} 4 \mathrm{~b} /$ G4 values measured with the G4 binder alone and in the presence of four additional compounds was found (Figure 2A,B and Table S2). This behavior was also maintained when testing one good G4 binder mixed with 320 druglike compounds from a commercial library: the MS spectra obtained in the presence of 4 or 320 compounds were almost superimposable (compare Figure $2 \mathrm{~B}, \mathrm{C}$ ), and the $\mathrm{G} 4 \mathrm{~b} / \mathrm{G} 4$ ratio only minimally decreased (Table S2). These data indicate the possibility to screen as far as 320 compounds at a time in a single tube and detect G4 binders with our AUF-MS protocol.

Library Screening against Two HIV-1 LTR G4s. We applied this method to test a library of 40000 druglike compounds for their binding to LTR-IV and LTR-III (Table S1). The 320 compounds in each round of analysis were randomly chosen from the commercial library plate. Considering only the hits with (G4b/G4)\% > 10, we found two compounds binding LTR-IV (F18 and K23) and two LTR-III (J20 and N13) (Table 1, Figures 3A, and S8-S16). J20 stood out as the compound with the highest (G4b/G4)\% value. Univocal identification of the binding compounds was performed by singularly testing all molecules that, within the 320 compounds present in the test tube, had MW corresponding to the observed mass shift (Table 1 and Figures S17-S20). The four hits were able to stabilize their target (i.e., LTR-III for J20 and N13, LTR-IV for F18 and N13) as assessed by CD (Table 1, Figures S21, and S22). J20 was the best stabilizing compound $\left(\Delta T_{\mathrm{m}}\right.$ (LTR-III) $3.9 \pm 0.5^{\circ} \mathrm{C}$, Table 1$)$ and the only one that largely modified the target G4 conformation upon interaction (Figures $3 \mathrm{~B}$ and S21). The nontarget G4s (i.e., LTR-IV and hTel for J20 and N13, LTRIII and hTel for F18 and N13) were not stabilized (Table 1, Figures S23, and S24), indicating that the hits were specifically identified toward their target by the AUF-MS method. To measure binding affinity, we first assessed by CD if biotinylation of the oligonucleotides to be used in SPR analysis modified their conformation: we observed changes both in spectra profiles and intensities (Figure S25). Thus, to measure binding affinity with the unmodified oligonucleotides, we employed ESI-MS analysis, as previously described. ${ }^{41} \mathrm{~J} 20$ binding affinity $\left(K_{\mathrm{D}}\right)$ to LTR-III measured at three compound concentrations and at 1:1 and 2:1 compound:DNA ratios was $11.1 \pm 1.3 \mu \mathrm{M}$ (Figure S26). In contrast, no binding was observed with any of the other test G4-forming and control sequences (Figure S27). These data confirm the specific interaction of J20 with LTR-III and the efficiency of the stringent conditions used in the screening.

G-Quadruplex Structure Validation in MS Buffer. Before trying to improve compound binding to LTR-III, we confirmed LTR-III G4 conformation in MS buffer by ${ }^{1} \mathrm{H}$ NMR. We recorded the ${ }^{1} \mathrm{H}$ NMR spectra of the imino protons in both MS buffer ${ }^{32}$ and $\mathrm{K}^{+}$buffer, with the latter previously used for structural assignment ${ }^{36}$ (Figure S28). Although MS buffer is clearly not the best choice for ${ }^{1} \mathrm{H}$ NMR analysis, comparison between the two spectra was possible. MS buffer spectrum in Figure S28A exhibited imino protons for all of the Gs except for G17 and G25 (see the embedded cartoon for Gnumbering). ${ }^{36}$ Although with lower resolution, the MS buffer imino protons exhibited almost identical chemical shifts to those recorded in $\mathrm{K}^{+}$buffer (Figure S28B). Additionally, a broader signal centered at $\sim 11 \mathrm{ppm}$, which included G17 and G25 imino protons, likely suggested the existence of one or more unresolved additional G4 conformations of an overall estimated abundance of $15 \%$, in MS buffer. These NMR data indicate that the LTR-III G4 structure previously published ${ }^{36}$ is largely preserved $(\sim 85 \%)$ in MS buffer.

Characterization of Improved LTR-III G4 Ligands. To increase J20 binding properties, we looked for available analogues. Three molecules sharing the same diethylaminomethyl, pyrazolo[ $[1,5-a]$ pyrimidine and carboxamide moieties, 

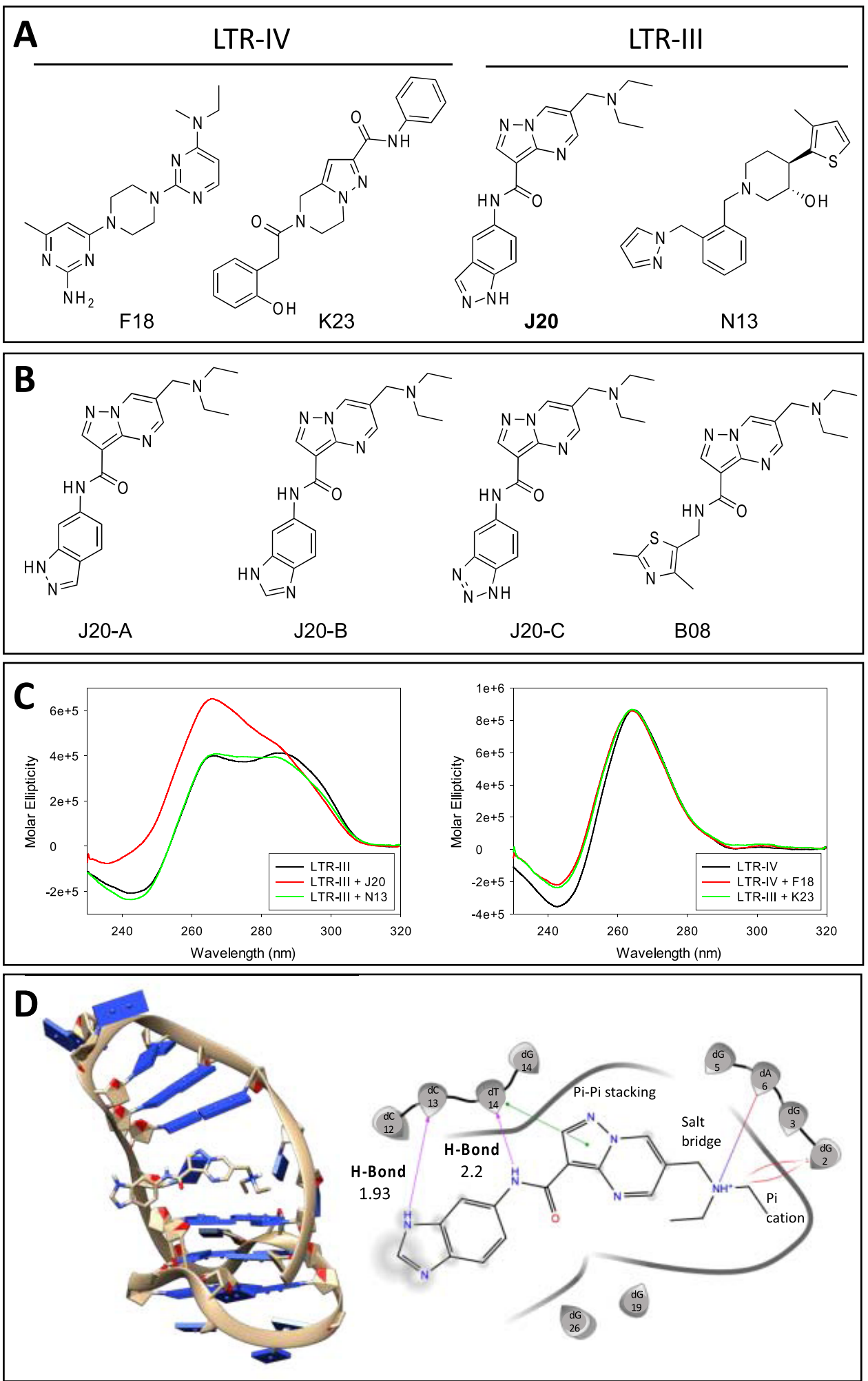

Figure 3. Structures and binding properties of hits from the AUF-MS HTS of 40000 druglike molecules. (A) Chemical structures of the hits obtained against LTR-IV and LTR-III G4s, as indicated. (B) J20 structural analogues, and negative control B08. (C) CD spectra of LTR-III and LTR-IV alone (left and right, respectively) and in the presence of their respective AUF-MS hits. (D) Molecular modeling analysis of J20-B toward LTR-III G4: best pose structure (left), structure and interaction mode (right).

while displaying a modified indazole group were identified in databases and purchased: J20-A carrying an indazole isomer, J20-B carrying a benzoimidazole, and J20-C carrying a benzotriazole (Figure 3B). The J20 analogues showed improved binding toward LTR-III G4 (G4b/G4 up to 2.4- folds and $\Delta T_{\mathrm{m}}$ up to $1.5^{\circ} \mathrm{C}$ higher than that of J20), while they were unable to bind and stabilize hTel G4 (Figures S29-S31). To determine the structural features that improved LTR-III binding, we performed molecular modeling of the three best ligands (J20-A, J20-B, J20-C) and one negative control, i.e., a 
chemical analogue (B08) present within the 40000 compounds of the library, sharing the same pyrazolopyrimidine core with $\mathrm{J} 20$ and not detected during the screening (Figure 3B). The induced-fit docking simulations provided a set of G4ligand conformers, ranked according to binding free energies (Glide score). J20-B formed the most stable complex with LTR-III (score -7.73, Figure 3D), while J20-A and J20-C displayed slightly lower scores $(-7.11$ and -7.12 , respectively). The binding pocket for all of the investigated ligands was the G4/stem-loop junction, a key structural feature of the LTR-III G4 $\operatorname{target}^{36}$ (Figure S32). Hydrogen bonding represented the key interaction that differentiated ligand binding (Figures 3D and S32).

The ability of J20 and J20-B, which displayed the best solubility in water, to stabilize their LTR-III G4 target in the presence of the nontarget LTR-IV G4 was assessed by Taq polymerase stop assay. We used an extended LTR sequence (LTR-III + IV), where both LTR-III and LTR-IV G4-forming G-tracts are present (Table S1). This sequence stalls polymerase progression at LTR-III in the presence of $\mathrm{K}^{+}$, whereas general G4-ligands induce stops at both LTR-III and LTR-IV. ${ }^{10}$ The two compounds were also assessed against hTel G4 and a control sequence unable to form G4 (Table S1). Both J20 and J20-B increased polymerase stop at LTR-III, while no stop was observed at LTR-IV (Figure 4A). J20-B displayed higher activity than J20 (Figure 4A, compare lanes 4 and 5 with lanes 2 and 3, LTR-III + IV); both J20 and J20-B were active against the LTR sequence only (Figure 4A, non-G4 control, hTel). B08 was inactive against all tested sequences (Figure 4A, lanes 6 and 7). These data fully agree and thus confirm the previous MS, CD, and molecular modeling data: J20-B is more potent than J20 and both stabilize LTR-III, while they are inactive on LTR-IV and hTel G4s.

After assessing that J20 did not bind to dsDNA and ssDNA (Figure S27), we proceeded to in-cell analysis. Stabilization of LTR G4s reduces HIV-1 LTR promoter activity, as previously demonstrated with other general G4 ligands. ${ }^{10,43,44}$ To test if J20/J20-B also presented this feature, we set up a luciferase reporter assay in HEK $293 \mathrm{~T}$ cells using the LTR-Luc plasmid containing the full-length HIV-1 LTR promoter. ${ }^{10,35} \mathrm{~J} 20$ and J20-B were able to reduce LTR activity up to 63 and $60 \%$, respectively, of the untreated control, while B08 was inactive. All three compounds were not cytotoxic (Figure 4B). The compounds' antiviral properties were next measured against HIV-1 (NL4-3 strain) in TMZ-bl reporter cells, concurrently to cell viability by MTT on the same uninfected cells. J20-B showed good antiviral activity $\left(\mathrm{EC}_{50} 5.4 \mu \mathrm{M}\right)$; J20 was 3 -fold less active $\left(\mathrm{EC}_{50} 17.2 \mu \mathrm{M}\right)$ (Figure $4 \mathrm{C}$ ); B08, tested at 10 and $20 \mu \mathrm{M}$, was inactive. All three compounds were not cytotoxic $\left(\mathrm{CC}_{50}>20 \mu \mathrm{M}\right)$ (Figure $4 \mathrm{C}$ ); thus, selectivity indexes were $>3.7$ and $>1.2$ for J20-B and J20, respectively.

\section{DISCUSSION AND CONCLUSIONS}

The possibility to screen the millions of available compounds against the G4 target of choice with fast and inexpensive methods would increase the possibility of finding the "perfect" selective and druglike ligands by serendipity, rather than rational design based on structure and sequence selectivity. ${ }^{18}$ HTS represents a mainstay of drug discovery. Most of the current HTS methods rely on fluorescence, which is modified upon complex formation as in the fluorescence resonance energy transfer (FRET) assay ${ }^{45,46}$ or used to identify complex location in microarrays. ${ }^{47,48}$ The main limitation in these

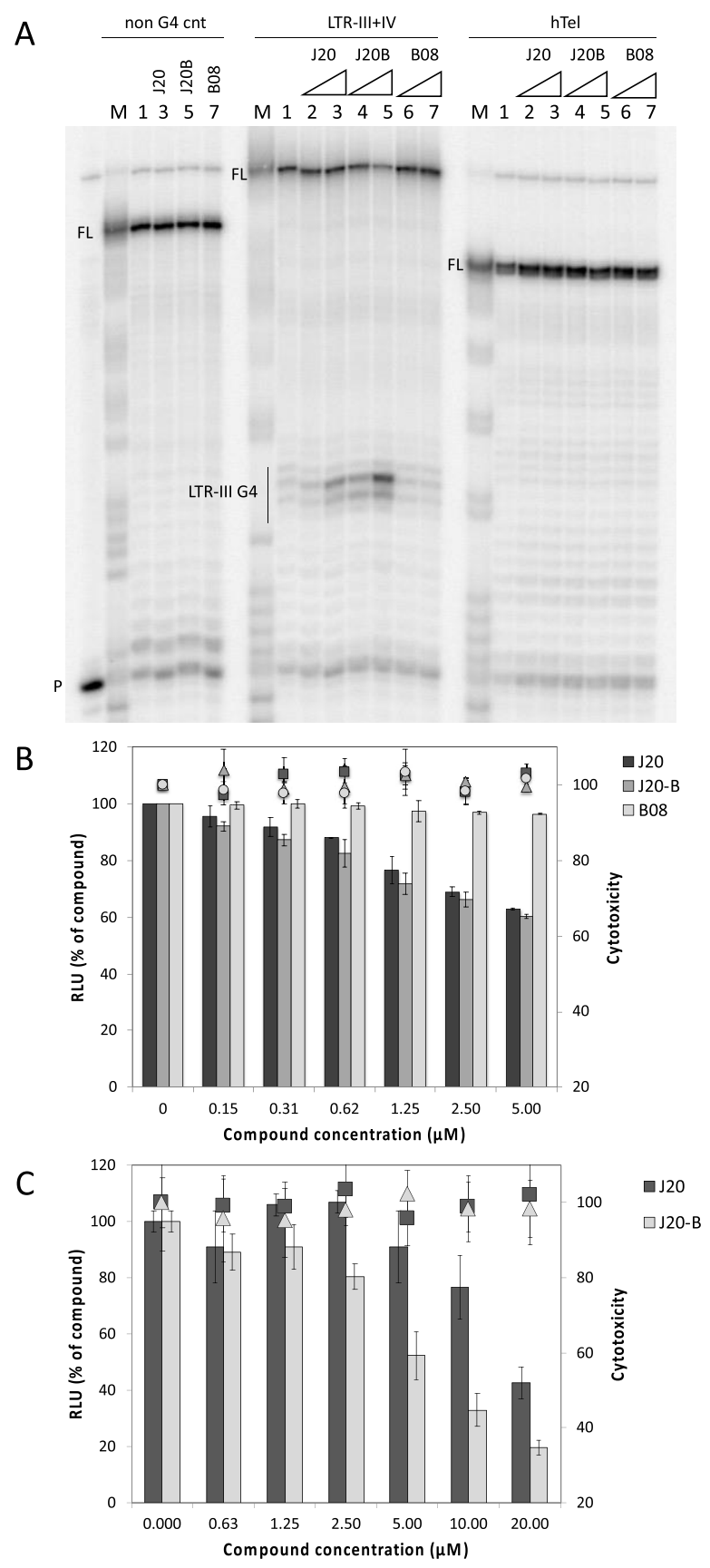

Figure 4. In vitro and in cell activity of J20 and J20-B. (A). Image of a typical Taq polymerase stop assay: LTR-III + IV and hTel templates were amplified by Taq polymerase in $20 \mathrm{mM} \mathrm{KCl}$, alone (lanes 1) or with increasing concentrations $(1-4 \mu \mathrm{M})$ of $\mathrm{J} 20$ (lanes 2 and 3), J20B (lanes 4 and 5), B08 (lanes 6 and 7). A template (non-G4 cnt) unable to fold into $\mathrm{G} 4$ was used as a negative control in $20 \mathrm{mM} \mathrm{KCl}$, alone (lane 1) or with $4 \mu \mathrm{M}$ of J20 (lane 3), J20B (lane 5), and B08 (lane 7). Lane P: unreacted labeled primer. Lane M: Maxam\&Gilbert marker. FL: full-length amplicon. The vertical bar indicates LTR-IIIspecific polymerase stops. (B) Compounds' effect on cell viability and on LTR promoter activity. Luciferase expression of HIV-1 LTR normalized to total protein content (RLU = relative luciferase units, bar graph, left $Y$ axis) and MTT assay (symbol graph, right $Y$ axis) in HEK $293 \mathrm{~T}$ cells in the presence of the indicated compounds. (C) J20/J20-B antiviral activity against HIV-1 NL4-3 in TZM-bl cells. Antiviral activity (RLU) was measured in infected cells (bar graph, left $Y$ axis), while cell viability was obtained in parallel by MTT assay in uninfected cells (symbol graph, right $Y$ axis). 
methods is the need to modify the interacting molecules with fluorophores or through attachment to chip surfaces that may alter or mask interacting sites. Displacement methods overcome this issue ${ }^{49}$ but maintain the need to singularly test each compound. HTS approaches usually yield high rates of falsenegative and, most importantly, false-positive hits that may lead to thousands of compounds to be tested in secondary assays, with consequent waste of time and finances. ${ }^{50}$

Here, we developed an AUF-MS HTS method that does not need prior modification of the interacting molecules and affords the concurrent testing of hundreds of molecules in the same test tube for the efficient and fast detection of G4 ligands. We have opted for the direct analysis of G4/ligand complexes (direct method) over detection of bound molecules released from the target (indirect method) because the latter requires initial complete removal of the unbound compounds, which is often not sufficiently effective and thus represents a major source of false-positive hits. ${ }^{51}$ Moreover, if the compounds do not ionize well when released from the target, they may remain under- or undetected by MS. In contrast, the direct method relies on transfer in the gas phase of the intact target/ligand complex and on detection of the mass shift compared to the mass of the target biomolecule. To analyze the complexes by MS, we employed our optimized buffer that allows MS detection of intact G4s without affecting their folding and ligand binding. ${ }^{32}$ In fact, CD-measured stabilization of G4s by known ligands in this MS buffer showed minimal differences with the buffer normally used in CD analysis. This strategy offers several advantages: (1) reduction of false-positive hits: the unbound molecules were removed and only ligands that were efficiently bound to the G4 target were detected. Indeed, screening of 40000 compounds by this method provided only two hits per G4 target. The low number of positive hits $(0.005 \%)$ is not completely surprising because similar hit rates were reported for other AUF-MS-based screening (0.02\% in ref 52 and $0.001 \%$ in ref 53) and the screened library displays druglike chemical properties and thus lacks the extended aromatic planar moiety that is generally required for efficient G4 recognition. ${ }^{54}$ (2) Reduction of false-negative hits since MS ionization settings were optimized for the target G4 and thus even nonionizing molecules bound to the G4 could be revealed. (3) Easy analysis since MS settings did not need to be varied on the basis of the ligand chemical properties. (4) Highly increased analysis speed since the preliminary removal of unbound molecules allowed us to test in a single tube several hundreds of molecules at a time. We have shown that the large amount of nonbinding compounds did not hinder or decrease binding affinity (see G4b/G4 in Table 1). The 40000 -compound library was tested in as low as 125 cycles, and libraries of 300-400 compounds can be tested in one single tube and in just one cycle of our HTS protocol (Figure $1)$. Note that (1) because of the washing steps, the retrieved hits will depend on both the binding affinity and dissociation rate of the complex, it is advisable to maintain the wash time constant to avoid biases among test compound mixes, and (2) the presence of a compound with high affinity for the target may hide weaker binders. Therefore, once a strong ligand is identified, repetition of the experiment without it is recommended to highlight possible additional weaker ligands. The hundreds of compounds present in the test tube could in principle interact among them and prevent their binding to the target. This effect was possibly the cause of the higher binding observed for J20 and N13 to their target LTR-III G4 when tested alone vs when tested in the 320-compound mix. However, the interaction among compounds would effectively compete and thus hinder the binding to the target only if the affinity among compounds was in the same range as that toward the target, i.e., $K_{\mathrm{D}} \leq 10 \mu \mathrm{M}$. We expect this type of interaction among tested compounds to be limited in number.

The best acting compounds identified here, i.e., J20 and its structural isomer J20-B, make a totally new class of G4 ligands with a low MW (363 Da) and no large aromatic surface. Our molecular docking simulation suggests that they fit and interact with residues in a binding pocket formed between the G4 upper planar surface and the stem loop of LTR-III G4. These results are mainly of qualitative nature, since while established with known and well-characterized poses in G4-ligand complexes, alternative docking configurations and solutions are still possible. In addition, Glide has been developed mainly for protein targets. To thoroughly sample alternative configurations for the complexes, long-time-scale MD simulations and enhanced sampling will be necessary. Notwithstanding these limitations, the docking analysis, along with binding and stabilization analysis, allowed us to gather basic structure-activity relationship (SAR) data: the indole moiety is necessary, since substitution with a thiazole group removes any activity; the position of the nitrogen atom in the pyrazole of the indole defines the potency of the compound activity; the 6-((diethylamino)methyl)pyrazolo[1,5-a]pyrimidine-3-carboxamide region is required for activity. The peculiar binding mode, lacking extensive interaction with the G-quartet, is likely at the basis of the observed low LTR-III G4 stabilization. The antiviral effect attests to a significant alteration of the target in the biological system, possibly mediated by interference with LTR interacting proteins.

G4s have been reported to be associated with transcription regulation $^{55,56}$ and to be present essentially in all living beings spanning from mammals to invertebrates, from plants to microorganisms. ${ }^{1,7,8,57,58}$ They have also been reported in the SARS-CoV-2 that is causing the current COVID-19 epidemics. ${ }^{59}$ We anticipate that the availability of an ultrafast screening method to identify druglike molecules that bind to relevant G4s may highly speed G4-based research and related therapies.

\section{ASSOCIATED CONTENT}

\section{SI Supporting Information}

The Supporting Information is available free of charge at https://pubs.acs.org/doi/10.1021/acs.analchem.0c04106.

Additional materials, CD, MS, NMR, and molecular docking analysis (PDF)

Molecular formula strings and properties of the 40000 library compounds (XLS)

\section{AUTHOR INFORMATION}

\section{Corresponding Author}

Sara N. Richter - Department of Molecular Medicine,

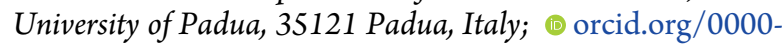
0002-5446-9029; Email: sara.richter@unipd.it

\author{
Authors \\ Matteo Scalabrin - Department of Molecular Medicine, \\ University of Padua, 35121 Padua, Italy
}


Matteo Nadai - Department of Molecular Medicine,

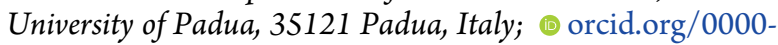
0003-3831-4892

Martina Tassinari - Department of Molecular Medicine,

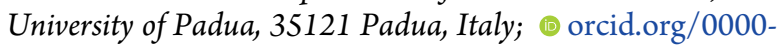
0002-3965-9334

Sara Lago - Department of Molecular Medicine, University of Padua, 35121 Padua, Italy; 10 orcid.org/0000-0001-5179$550 \mathrm{X}$

Filippo Doria - Department of Chemistry, University of Pavia, 27100 Pavia, Italy

Ilaria Frasson - Department of Molecular Medicine, University of Padua, 35121 Padua, Italy

Mauro Freccero - Department of Chemistry, University of Pavia, 27100 Pavia, Italy; ㅇo orcid.org/0000-0002-74381526

Complete contact information is available at:

https://pubs.acs.org/10.1021/acs.analchem.0c04106

\section{Author Contributions}

${ }^{\S}$ M.S. and M.N. contributed equally to this work. The manuscript was written through contributions of all authors. All authors have given approval to the final version of the manuscript.

\section{Notes}

The authors declare no competing financial interest.

\section{ACKNOWLEDGMENTS}

This work was supported by the European Research Council [ERC Consolidator grant 615879] and the Bill and Melinda Gates Foundation [grant numbers OPP1035881 and OPP1097238]. The authors thank Prof. Giorgio Colombo and Matteo Castelli at the University of Pavia for the use of Schrondinger 2019-3 and Glide 6.9 softwares.

\section{REFERENCES}

(1) Hänsel-Hertsch, R.; Di Antonio, M.; Balasubramanian, S. Nat. Rev. Mol. Cell Biol. 2017, 18, 279-284.

(2) Tarsounas, M.; Tijsterman, M. J. Mol. Biol. 2013, 425, 47824789.

(3) Huppert, J. L.; Balasubramanian, S. Nucleic Acids Res. 2007, 35, 406-413.

(4) Cimino-Reale, G.; Zaffaroni, N.; Folini, M. Curr. Pharm. Des. 2017, 22, 6612-6624.

(5) Haeusler, A. R.; Donnelly, C. J.; Periz, G.; Simko, E. A. J.; Shaw, P. G.; Kim, M. S.; Maragakis, N. J.; Troncoso, J. C.; Pandey, A.; Sattler, R.; Rothstein, J. D.; Wang, J. Nature 2014, 507, 195-200.

(6) Harris, L. M.; Merrick, C. J. PLoS Pathog. 2015, 11, No. e1004562.

(7) Saad, M.; Guédin, A.; Amor, S.; Bedrat, A.; Tourasse, N. J.; Fayyad-Kazan, H.; Pratviel, G.; Lacroix, L.; Mergny, J.-L. Nucleic Acids Res. 2019, 47, 4363-4374.

(8) Ruggiero, E.; Richter, S. N. Nucleic Acids Res. 2018, 46, 32703283.

(9) Heeney, J. L.; Dalgleish, A. G.; Weiss, R. A. Science 2006, 313, 462-466.

(10) Perrone, R.; Nadai, M.; Frasson, I.; Poe, J. A.; Butovskaya, E.; Smithgall, T. E.; Palumbo, M.; Palù, G.; Richter, S. N. J. Med. Chem. 2013, 56, 6521-6530.

(11) Piekna-Przybylska, D.; Sullivan, M. A.; Sharma, G.; Bambara, R. A. Biochemistry 2014, 53, 2581-2593.

(12) Tosoni, E.; Frasson, I.; Scalabrin, M.; Perrone, R.; Butovskaya, E.; Nadai, M.; Palù, G.; Fabris, D.; Richter, S. N. Nucleic Acids Res. 2015, 43, 8884-8897.
(13) Scalabrin, M.; Frasson, I.; Ruggiero, E.; Perrone, R.; Tosoni, E.; Lago, S.; Tassinari, M.; Palù, G.; Richter, S. N. Sci. Rep. 2017, 7, No. 45244.

(14) Balasubramanian, S.; Neidle, S. Curr. Opin. Chem. Biol. 2009, 13, 345-353.

(15) Nadai, M.; Doria, F.; Scalabrin, M.; Pirota, V.; Grande, V.; Bergamaschi, G.; Amendola, V.; Winnerdy, F. R.; Phan, A. T.; Richter, S. N.; Freccero, M. J. Am. Chem. Soc. 2018, 140, 14528-14532.

(16) Carvalho, J.; Mergny, J.-L.; Salgado, G. F.; Queiroz, J. A.; Cruz, C. Trends Mol. Med. 2020, 26, 848-861.

(17) Pirota, V.; Nadai, M.; Doria, F.; Richter, S. Molecules 2019, 24, 426.

(18) Tassinari, M.; Zuffo, M.; Nadai, M.; Pirota, V.; Sevilla Montalvo, A. C.; Doria, F.; Freccero, M.; Richter, S. N. Nucleic Acids Res. 2020, 48, 4627-4642.

(19) Felsenstein, K. M.; Saunders, L. B.; Simmons, J. K.; Leon, E.; Calabrese, D. R.; Zhang, S.; Michalowski, A.; Gareiss, P.; Mock, B. A.; Schneekloth, J. S. ACS Chem. Biol. 2016, 11, 139-148.

(20) Calabrese, D. R.; Chen, X.; Leon, E. C.; Gaikwad, S. M.; Phyo, Z.; Hewitt, W. M.; Alden, S.; Hilimire, T. A.; He, F.; Michalowski, A. M.; Simmons, J. K.; Saunders, L. B.; Zhang, S.; Connors, D.; Walters, K. J.; Mock, B. A.; Schneekloth, J. S. Nat. Commun. 2018, 9, No. 4229.

(21) Deiana, M.; Chand, K.; Jamroskovic, J.; Das, R. N.; Obi, I.; Chorell, E.; Sabouri, N. Nanoscale 2020, 12, 12950-12957.

(22) Zhai, Q.; Gao, C.; Ding, J.; Zhang, Y.; Islam, B.; Lan, W.; Hou, H.; Deng, H.; Li, J.; Hu, Z.; Mohamed, H. I.; Xu, S.; Cao, C.; Haider, S. M.; Wei, D. Nucleic Acids Res. 2019, 47, 2190-2204.

(23) Dutta, D.; Debnath, M.; Müller, D.; Paul, R.; Das, T.; Bessi, I.; Schwalbe, H.; Dash, J. Nucleic Acids Res. 2018, 46, 5355-5365.

(24) Hu, M.-H.; Wu, T.-Y.; Huang, Q.; Jin, G. Nucleic Acids Res. 2019, 47, 10529-10542.

(25) Fenn, J.; Mann, M.; Meng, C.; Wong, S.; Whitehouse, C. Science 1989, 246, 64-71.

(26) Gabelica, V.; Galic, N.; Rosu, F.; Houssier, C.; De Pauw, E. J. Mass Spectrom. 2003, 38, 491-501.

(27) Sannes-Lowery, K. A.; Griffey, R. H.; Hofstadler, S. A. Anal. Biochem. 2000, 280, 264-271.

(28) Cheng, X.; Chen, R.; Bruce, J. E.; Schwartz, B. L.; Anderson, G. A.; Hofstadler, S. A.; Gale, D. C.; Smith, R. D.; Gao, J.; Sigal, G. B.; Mammen, M.; Whitesides, G. M. J. Am. Chem. Soc. 1995, 117, 88598860.

(29) Marchand, A.; Gabelica, V. J. Am. Soc. Mass Spectrom. 2014, 25, $1146-1154$.

(30) Smargiasso, N.; Rosu, F.; Hsia, W.; Colson, P.; Baker, E. S.; Bowers, M. T.; De Pauw, E.; Gabelica, V. J. Am. Chem. Soc. 2008, 130, 10208-10216.

(31) Vorlíčková, M.; Bednárová, K.; Kypr, J. Biopolymers 2006, 82, 253-260.

(32) Scalabrin, M.; Palumbo, M.; Richter, S. N. Anal. Chem. 2017, $89,8632-8637$.

(33) Marchand, A.; Rosu, F.; Zenobi, R.; Gabelica, V. J. Am. Chem. Soc. 2018, 140, 12553-12565.

(34) Marchand, A.; Erie Gabelica, V. Nucleic Acids Res. 2016, 44, 10999-11012.

(35) De Nicola, B.; Lech, C. J.; Heddi, B.; Regmi, S.; Frasson, I.; Perrone, R.; Richter, S. N.; Phan, A. T. Nucleic Acids Res. 2016, 44, 6442-6451.

(36) Butovskaya, E.; Heddi, B.; Bakalar, B.; Richter, S. N.; Phan, A. T. J. Am. Chem. Soc. 2018, 140, 13654-13662.

(37) Doria, F.; Nadai, M.; Folini, M.; Di Antonio, M.; Germani, L.; Percivalle, C.; Sissi, C.; Zaffaroni, N.; Alcaro, S.; Artese, A.; Richter, S. N.; Freccero, M. Org. Biomol. Chem. 2012, 10, 2798-2806.

(38) Nadai, M.; Doria, F.; Di Antonio, M.; Sattin, G.; Germani, L.; Percivalle, C.; Palumbo, M.; Richter, S. N.; Freccero, M. Biochimie 2011, 93, 1328-1340.

(39) Doria, F.; Nadai, M.; Costa, G.; Sattin, G.; Gallati, C.; Bergamaschi, G.; Moraca, F.; Alcaro, S.; Freccero, M.; Richter, S. N. Eur. J. Org. Chem 2016, 2016, 4824-4833.

(40) Greenfield, N. J. Nat. Protoc. 2006, 1, 2527-2535. 
(41) Lombardo, C. M.; Martínez, I. S.; Haider, S.; Gabelica, V.; De Pauw, E.; Moses, J. E.; Neidle, S. Chem. Commun. 2010, 46, 91169118.

(42) Prudent, R.; Annis, D. A.; Dandliker, P. J.; Ortholand, J.-Y.; Roche, D. Nat. Rev. Chem. 2021, 5, 62-71.

(43) Perrone, R.; Butovskaya, E.; Daelemans, D.; Palu, G.; Pannecouque, C.; Richter, S. N. J. Antimicrob. Chemother. 2014, 69, $3248-3258$.

(44) Perrone, R.; Doria, F.; Butovskaya, E.; Frasson, I.; Botti, S.; Scalabrin, M.; Lago, S.; Grande, V.; Nadai, M.; Freccero, M.; Richter, S. N. J. Med. Chem. 2015, 58, 9639-9652.

(45) Renčiuk, D.; Zhou, J.; Beaurepaire, L.; Guédin, A.; Bourdoncle, A.; Mergny, J.-L. Methods 2012, 57, 122-128.

(46) Wang, K.; Flaherty, D. P.; Chen, L.; Yang, D. Methods Mol. Biol. 2019, 2035, 323-331.

(47) Connelly, C. M.; Abulwerdi, F. A.; Schneekloth, J. S. Discovery of RNA Binding Small Molecules Using Small Molecule Microarrays. In Methods in Molecular Biology; NIH Public Access: New York, NY, 2017; Vol. 1518, pp 157-175.

(48) Ray, S.; Tillo, D.; Boer, R. E.; Assad, N.; Barshai, M.; Wu, G.; Orenstein, Y.; Yang, D.; Schneekloth, J. S.; Vinson, C. ACS Chem. Biol. 2020, 15, 925-935.

(49) Largy, E.; Hamon, F.; Teulade-Fichou, M.-P. Anal. Bioanal. Chem. 2011, 400, 3419-3427.

(50) Sink, R.; Gobec, S.; Pečar, S.; Zega, A. Curr. Med. Chem. 2010, 17, 4231-4255.

(51) Sun, Y.; Gu, C.; Liu, X.; Liang, W.; Yao, P.; Bolton, J. L.; Van Breemen, R. B. J. Am. Soc. Mass Spectrom. 2005, 16, 271-279.

(52) Comess, K. M.; Schurdak, M. E.; Voorbach, M. J.; Coen, M.; Trumbull, J. D.; Yang, H.; Gao, L.; Tang, H.; Cheng, X.; Lerner, C. G.; Mccall, J. O.; Burns, D. J.; Beutel, B. A. J. Biomol. Screening 2006, $11,743-754$.

(53) Comess, K. M.; Sun, C.; Abad-Zapatero, C.; Goedken, E. R.; Gum, R. J.; Borhani, D. W.; Argiriadi, M.; Groebe, D. R.; Jia, Y.; Clampit, J. E.; Haasch, D. L.; Smith, H. T.; Wang, S.; Song, D.; Coen, M. L.; Cloutier, T. E.; Tang, H.; Cheng, X.; Quinn, C.; Liu, B.; Xin, Z.; Liu, G.; Fry, E. H.; Stoll, V.; Ng, T. I.; Banach, D.; Marcotte, D.; Burns, D. J.; Calderwood, D. J.; Hajduk, P. J. ACS Chem. Biol. 2011, 6, 234-244.

(54) Haider, S. M.; Neidle, S.; Parkinson, G. N. Biochimie 2011, 93, $1239-1251$.

(55) Hänsel-Hertsch, R.; Beraldi, D.; Lensing, S. V.; Marsico, G.; Zyner, K.; Parry, A.; Di Antonio, M.; Pike, J.; Kimura, H.; Narita, M.; Tannahill, D.; Balasubramanian, S. Nat. Genet. 2016, 48, 1267-1272.

(56) Varshney, D.; Spiegel, J.; Zyner, K.; Tannahill, D.; Balasubramanian, S. Nat. Rev. Mol. Cell Biol. 2020, 21, 459-474.

(57) Ding, Y.; Fleming, A. M.; Burrows, C. J. Sci. Rep. 2018, 8, No. 15679.

(58) Marsico, G.; Chambers, V. S.; Sahakyan, A. B.; McCauley, P.; Boutell, J. M.; Antonio, M. D.; Balasubramanian, S. Nucleic Acids Res. 2019, 47, 3862-3874.

(59) Bartas, M.; Brázda, V.; Bohálová, N.; Cantara, A.; Volná, A.; Stachurová, T.; Malachová, K.; Jagelská, E. B.; Porubiaková, O.; Červeň, J.; Pečinka, P. Front. Microbiol. 2020, 11, No. 1583. 